\title{
Observation of wakefields in a beam-driven photonic band gap accelerating structure
}

\author{
C. Jing, ${ }^{1,2}$ F. Gao, ${ }^{1,2, *}$ S. Antipov, ${ }^{1}$ Z. Yusof,,${ }^{1}$ M. Conde,,${ }^{1}$ J. G. Power, ${ }^{1}$ P. Xu, ${ }^{3}$ S. Zheng, ${ }^{3}$ H. Chen, ${ }^{3}$ C. Tang,,${ }^{3}$ and W. Gai ${ }^{1}$ \\ ${ }^{1}$ High Energy Physics Division, Argonne National Laboratory, Argonne, Illinois 60439, USA \\ ${ }^{2}$ Euclid Techlabs LLC, Solon, Ohio 44139, USA \\ ${ }^{3}$ Department of Engineering Physics, Tsinghua University, Beijing, China \\ (Received 20 September 2009; published 23 December 2009)
}

\begin{abstract}
Wakefield excitation has been experimentally studied in a three-cell X-band standing wave photonic band gap (PBG) accelerating structure. Major monopole $\left(\mathrm{TM}_{01^{-}}\right.$and $\mathrm{TM}_{02}$-like $)$ and dipole $\left(\mathrm{TM}_{11^{-}}\right.$and $\mathrm{TM}_{12}$-like) modes were identified and characterized by precisely controlling the position of beam injection. The quality factor $Q$ of the dipole modes was measured to be $\sim 10$ times smaller than that of the accelerating mode. A charge sweep, up to $80 \mathrm{nC}$, has been performed, equivalent to $\sim 30 \mathrm{MV} / \mathrm{m}$ accelerating field on axis. A variable delay low charge witness bunch following a high charge drive bunch was used to calibrate the gradient in the PBG structure by measuring its maximum energy gain and loss. Experimental results agree well with numerical simulations.
\end{abstract}

DOI: 10.1103/PhysRevSTAB.12.121302

PACS numbers: 29.20.Ej

Photonic band gap (PBG) structures for particle acceleration have been intensively investigated since the concept was introduced more than a decade ago [1,2]. The capability of single mode confinement makes it very attractive for future particle accelerator designs which require large transverse deflecting mode suppression $[3,4]$. Metal, dielectric-based, and hybrid PBG structures have been investigated both theoretically and experimentally [5-7]. In particular, $35 \mathrm{MV} / \mathrm{m}$ accelerating gradient was successfully demonstrated in the beam test of a $17 \mathrm{GHz}$ externally powered PBG accelerating structure [8]. Another beam experiment intended to measure the wakefield excitation in the same structure without external rf power was also performed [9]. Wakefields including the monopole (accelerating) and dipole (deflecting) modes generated in a PBG accelerating structure by a beam have never been clearly characterized in a beam test, however. Some theoretical studies have been published $[10,11]$.

The PBG accelerating structure discussed here consists of a periodic array of metallic rods. Because of the periodicity of the structure, a range of frequency bands (band gaps) exists through which it is forbidden for electromagnetic radiation to propagate. In order to allow particles to move through the structure, one rod is removed from the lattice to create a beam channel. This removal creates a defect in the "crystal" where the accelerating mode will be confined. The lattice parameters can be chosen in such a way that neither the transverse modes nor the high order monopole modes are confined in the beam channel $[12,13]$. In the real structure, the number of rods is finite. This leads both to a decrease in the quality factor of the accelerating mode because of imperfect confinement in the channel and the possibility of trapping higher order modes (HOMs).

\footnotetext{
*Present address: National Light Source, Brookhaven National Laboratory, Upton, NY 11973.
}

However, with a proper design, the quality factor of HOMs is, in general, significantly lower than that of the accelerating mode [11]. Several methods have been proposed to attenuate HOMs in finite lattices, such as using microwave absorbers [5,14], replacing outer rods with absorbing dielectric rods [15], and adjusting the position of the rods in the lattice to increase the quality factor of the accelerating mode while reducing the quality factor of the transverse modes [11]. To implement a similar damping factor for the transverse modes in a conventional metal based accelerating structure, the geometry and the fabrication will be much more complicated $[3,4]$. In this paper, we report on a successful wakefield characterization in a beam test of an $\mathrm{X}$-band three-cell PBG accelerating structure, in which the (fundamental and high order) monopole modes and dipole modes were profiled by precisely varying the beam offset relative to the nominal beam axis of the structure. A wakefield acceleration experiment in a collinear drive and witness beam configuration was also performed to calibrate the accelerating gradient generated by an $80 \mathrm{nC}$ electron bunch.

Unless specially mentioned, all discussions in the paper are concentrated in the microwave frequency region. However, we should point out that several studies have been made on optical PBG accelerating structures as well $[16,17]$. The characteristics of the monopole and dipole modes in PBG accelerating structures are the same regardless of the frequency range. In addition to particle acceleration, the PBG structure can also be used in high power rf source design [18].

We designed the $11.424 \mathrm{GHz}$ externally powered traveling wave PBG accelerating structure using a twodimensional triangular lattice of copper rods between two copper plates [19]. The beam channel was formed by removing the center rod and drilling irises on the plates. The phase advance between every two plates is 120 degrees at $11.424 \mathrm{GHz}$, designed so that the fundamental mode is 
TABLE I. Designed parameters of the X-band traveling wave PBG accelerating structure.

\begin{tabular}{ll}
\hline \hline Geometric and accelerating parameters & Value \\
\hline Rod radius, $a$ & $1.64 \mathrm{~mm}$ \\
Spacing between rods, $b$ & $10.19 \mathrm{~mm}$ \\
Iris diameter & $9.61 \mathrm{~mm}$ \\
Iris thickness & $1.71 \mathrm{~mm}$ \\
Plate spacing & $7.04 \mathrm{~mm}$ \\
Synchronized frequency & $11.424 \mathrm{GHz}$ \\
of the accelerating mode & $(2 \pi / 3 \mathrm{mode})$ \\
Group velocity & $0.05 \mathrm{c}$ \\
$Q$ & 5461 \\
$r / Q$ & $10.5 \mathrm{k} \Omega / \mathrm{m}$ \\
\hline \hline
\end{tabular}

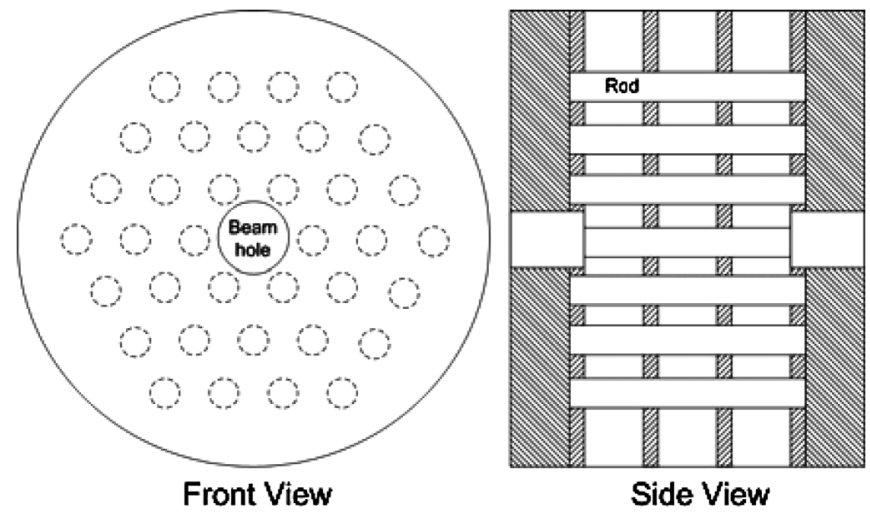

FIG. 1. Schematic drawings of the three-cell standing wave PBG structure used in the beam test.

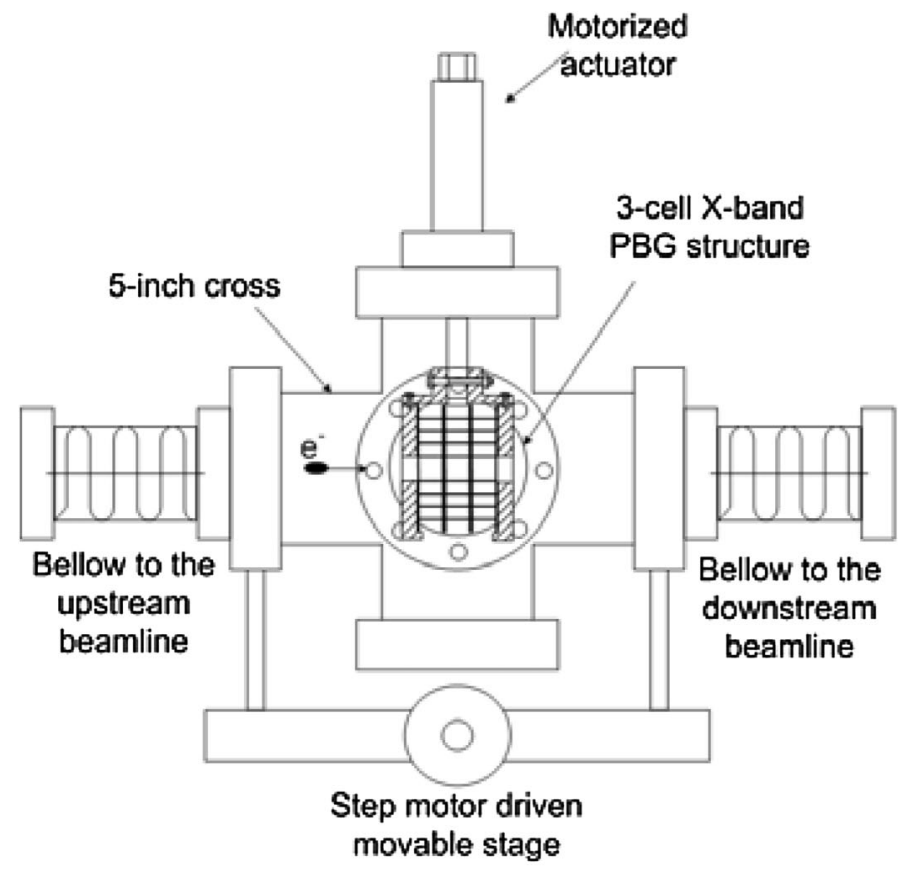

(a) synchronized with an ultrarelativistic beam. The major design parameters of the structure are summarized in Table I. Three cells have been prototyped using the electroforming technique. With the aim of experimentally studying the wakefield in the PBG structure, we assembled three PBG cells serving as a standing wave PBG structure (see Fig. 1). The fundamental monopole modes derived from the simulation are $11.1,11.31$, and $11.52 \mathrm{GHz}$, representing $0, \pi / 3$, and $2 \pi / 3$-like spatial modes, respectively. The frequency of each mode differs slightly from that in the traveling wave case because of the different boundary conditions. However, this did not affect the goal of this investigation in terms of monopole/dipole mode identification and characterization.

The wakefield experiment was performed at Argonne Wakefield Accelerator (AWA) facility located at Argonne National Laboratory (ANL), where a $1.3 \mathrm{GHz}$ photoinjector can provide $\sim 100 \mathrm{nC}$ charge in single bunch operation or several tens of $\mathrm{nC}$ per bunch in bunch train operation (charge varies with the bunch number per train) [20]. The energy of the electron bunch is boosted to $\sim 14 \mathrm{MeV}$ after one section of linac. The experimental setup is shown in Fig. 2. The PBG structure was mounted on a motorized actuator through a special fixture and housed inside a sixway vacuum cross. An ultrahigh vacuum (UHV) compatible coaxial rf probe was used to monitor the wakefield signal excited by the electron bunch. The probe was mounted on a 1.33 inch bellows on one port of the cross so that the pin tip of the rf probe can be moved in and out the PBG lattice if necessary. The whole cross, together with the probe, was mounted on top of a stepping motor-

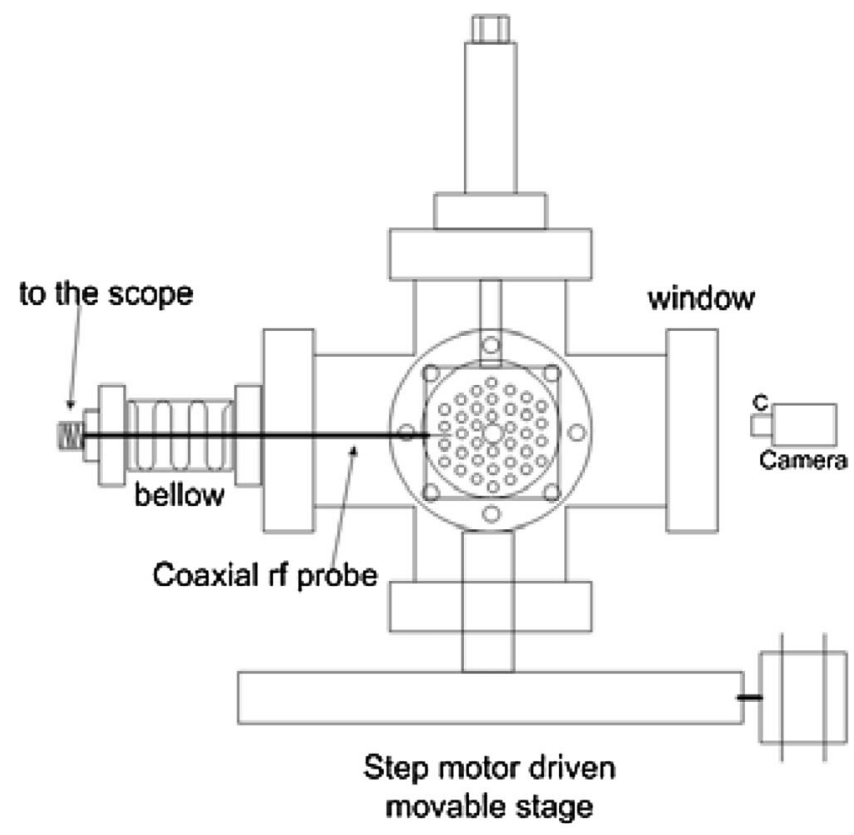

(b)

FIG. 2. Experimental setup in the AWA beam line: (a) side view; (b) front view. 
driven translation stage. Meanwhile, the cross was inserted into the AWA beam line through two 3-3/8 inch bellows on both ends, which was able to provide the mechanical flexibility for the cross to move a few $\mathrm{mm}$ transversely. This configuration would enable us to precisely control the beam offset in the PBG structure by remotely shifting the cross perpendicular to the beam axis via a stepping motor, ensuring that the position of the probe relative to the PBG structure was always fixed. Other diagnostics during the experiment included: (1) two inductive current transformers (ICTs) on both sides of the six-way cross to record the charge entering and exiting the structure; (2) phosphor screens on each side of the cross to monitor the transverse beam profile; (3) an energy spectrometer on the downstream end to measure the beam energy; (4) a CCD camera to detect any light from the possible rf breakdown of the PBG structure through the window on one port of the cross.

In the experiment, the rf signal of the excited wakefield in the PBG structure was captured by the probe and transmitted to a $16 \mathrm{GHz}$ digital oscilloscope $(50 \mathrm{Gs} / \mathrm{s}$ sampling rate). Figure 3(a) shows the power spectrum of a typical signal generated by a low charge $(\sim 0.5 \mathrm{nC})$, short ( $\sim 1.5 \mathrm{~mm}$ rms bunch length) off-axis electron bunch. The corresponding simulation of the electric field pattern for each mode is also plotted. The simulation was performed using CST Particle Studio ${ }^{\circledR}$. The experimental conditions were modeled closely, including the vacuum layer around the PBG structure and the outside metal chamber. With a proper choice of mesh size, the frequency of each mode in the simulation was reproduced within $\sim 5 \mathrm{MHz}$ of the experimental results. The lowest dipole mode $\left(\mathrm{TM}_{11}\right.$-like), has been identified at $14.46 \mathrm{GHz}$. The other significant modes shown in Fig. 3(a) include a higher order monopole mode $\left(\mathrm{TM}_{02}\right)$ at $14.88 \mathrm{GHz}$; and three high order dipole modes $\left(\mathrm{TM}_{12}\right.$-like), at around $15.27 \mathrm{GHz}$. Note that, in the spectrum plot, all modes, except for the three fundamental monopole modes, have broader widths. This means that in the time domain, the signals decay much faster than the monopole modes, indicating that $Q$ for all HOMs is lower. Mathematically, we can obtain the $Q$ of each mode by filtering them out in the frequency domain and transforming back to the time domain using the inverse fast-Fourier-transform (IFFT), followed by performing a decay curve fitting of the signal envelope. We should point out that the accuracy of $Q$ in this technique is limited by the frequency resolution of the signal and the mode spacing. In the experiment, the probe signals were recorded in a $400 \mathrm{~ns}$ time window, which gives us a $2.5 \mathrm{MHz}$ frequency resolution. As an example, also shown in Figs. 3(b) and 3(c), we calculated the $Q$ of the $2 \pi / 3$ mode at $11.55 \mathrm{GHz}$ to be $\sim 1400$ and $\sim 170$ for the $Q$ of the dipole mode at $14.46 \mathrm{GHz}$. This result agrees with some earlier works, for example, the bench measurement of a similar X-band PBG structure in Ref. [21]. It successfully demonstrates the intrinsic advantage of the PBG accelerating structure in terms of HOM suppression. Figure 4 shows the time-frequency representation of this probe signal captured when the beam is offset. The plot is generated by a short-time Fourier transformation that uses a $40 \mathrm{~ns}$ Hanning window shifting by 1 ns per step. We can clearly see that the fundamental monopole mode, particularly the $2 \pi / 3$ mode, oscillates over $150 \mathrm{~ns}$ while the dipole modes die down in $20 \mathrm{~ns}$.
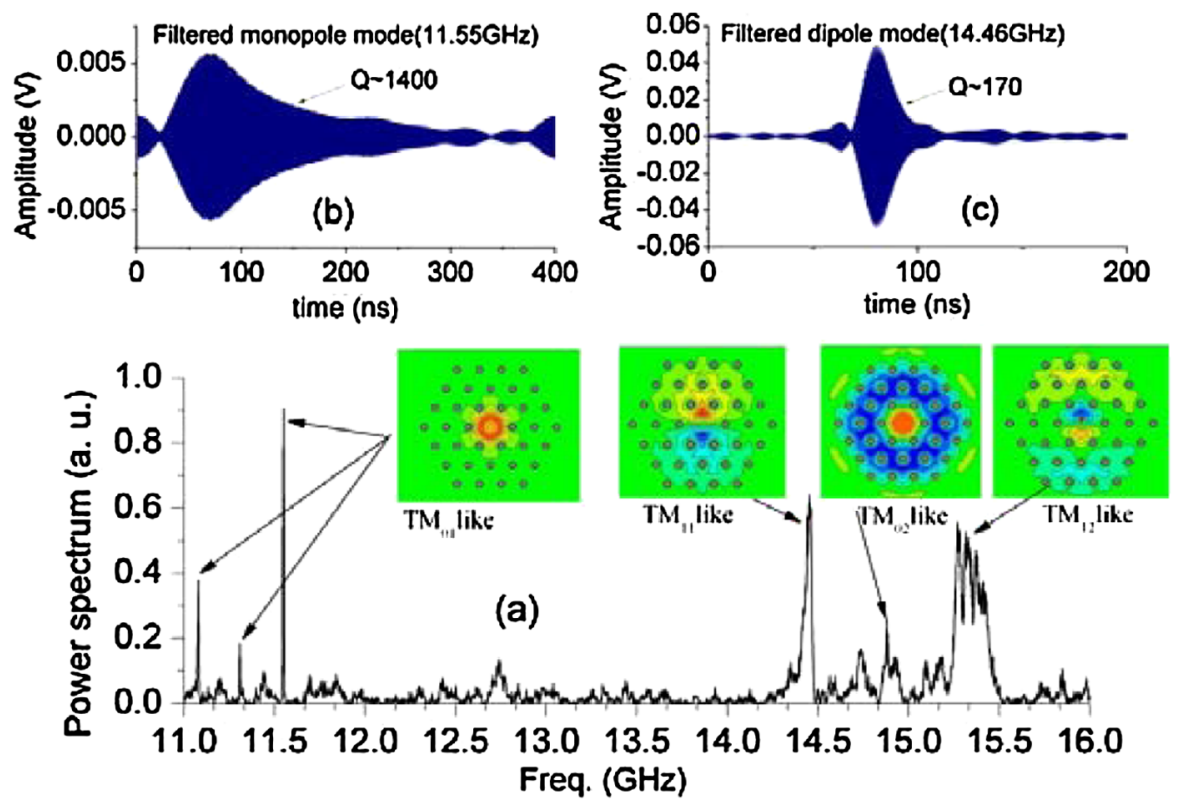

FIG. 3. (Color) (a) Power spectrum of the typical wakefield signal from an off-axis bunch captured by the rf probe and the simulated electrical field pattern for each major mode. (b) Signal of the filtered fundamental monopole mode (11.55 GHz). (c) Signal of the filtered lowest dipole mode $(14.46 \mathrm{GHz})$. 


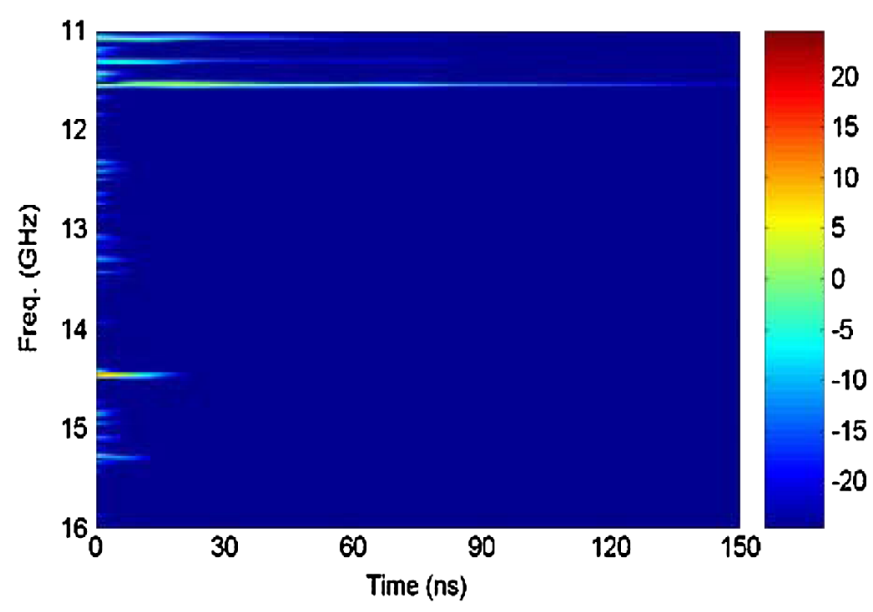

FIG. 4. (Color) Time-frequency analysis of the wakefield signal from an off-axis bunch captured by the rf probe.

In order to experimentally identify the major monopole and dipole modes using a different approach, we characterized the behavior of each mode by precisely controlling the electron bunch position across the beam iris of the PBG structure. This was implemented through the stepping motor-driven translation stage presented in Fig. 2. A single $\sim 0.5 \mathrm{nC}$ bunch was used to ensure that $100 \%$ of the beam passes through the PBG structure. We recorded the charge out of the structure and the probe signal simultaneously so that we could normalize the signal strength to the charge in the plots. Location of the minimum dipole signal, which represents the actual beam center, was slightly $(<1 \mathrm{~mm})$ away from the physical center of the beam line where we have indicated zero offset in the plots. The signal strength of the dipole modes was measured above zero because of the background noise. The measurement results are shown in Fig. 5. As we expect, the signal strength of three fundamental monopole modes is independent of the beam offset [see Fig. 5(a)]; but that of the dipole modes shows a very strong dependence [see Figs. 5(b) and 5(c)].

In addition, we tested this PBG structure using the unique high charge capability of the AWA beam line. Up to $80 \mathrm{nC}$ charge (single bunch) were transmitted through the structure, which was able to excite a gradient of $30 \mathrm{MV} / \mathrm{m}$ on axis based on the simulation. In order to experimentally calibrate the gradient, we performed a collinear wakefield acceleration test in which the energy gain/ loss of a low charge $(\sim 1 \mathrm{nC})$ witness bunch resulting from a high charge $(\sim 25 \mathrm{nC})$ drive bunch was measured. Both the drive and witness bunch were generated from the same $1.3 \mathrm{GHz}$ photoinjector, where their temporal separation were controlled through an optical delay. In the experiment, total $20 \mathrm{~mm}$ delay range was swept, which is equivalent to $\sim 300$ degrees of the rf phase change for the $11.5 \mathrm{GHz}$ signal. Energy loss of the witness bunch was clearly measured over the deceleration phase but the en-

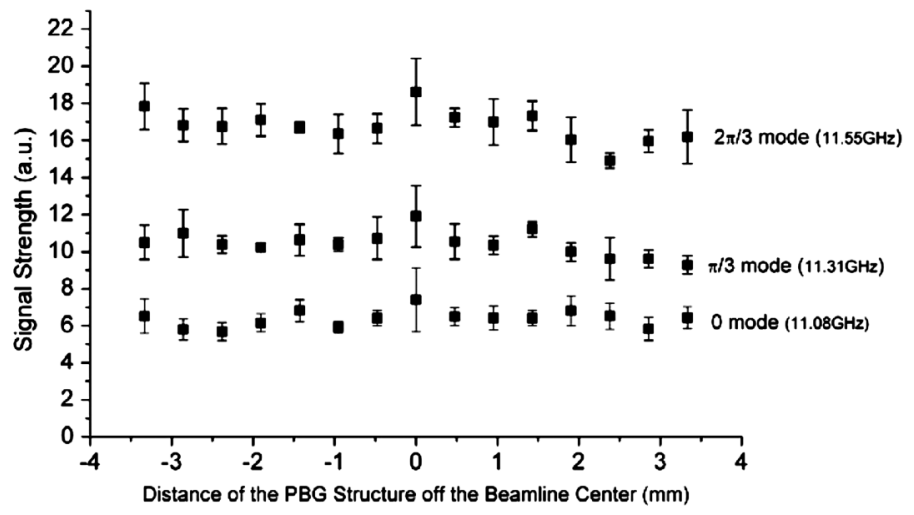

(a)

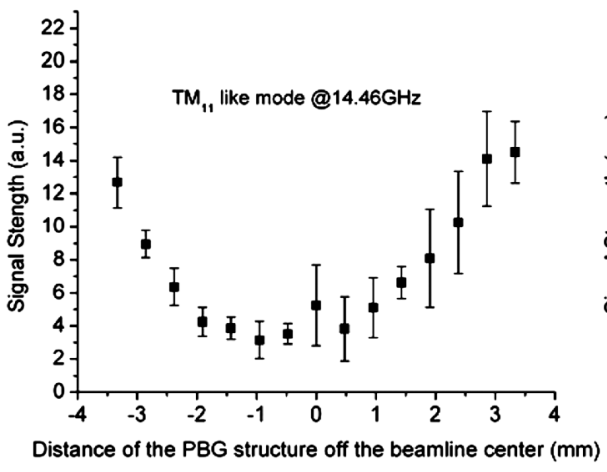

(b)

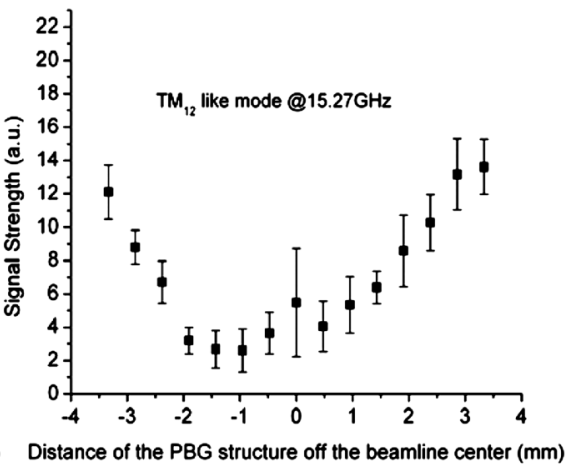

(c)

FIG. 5. Measured signal strengths for three fundamental monopole (a) and two dipole modes (b) and (c) with varying beam offset. 


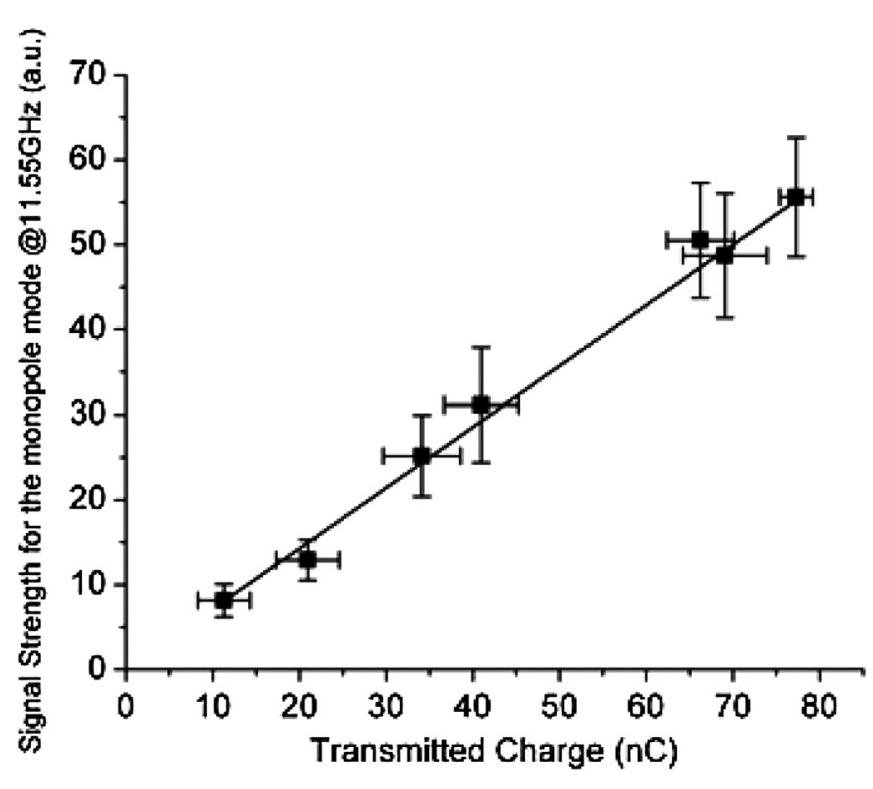

FIG. 6. Dependence of the signal strength of one monopole mode (obtained from the rf probe) on the beam charge. Referring to the MAFIA ${ }^{\circledR}$ simulation and the collinear wakefield acceleration measurement, $\sim 30 \mathrm{MV} / \mathrm{m}$ gradient on axis can be reached when an $80 \mathrm{nC}$ bunch is transmitted through the structure.

ergy gain could not be measured because of the large energy spread in the acceleration phase. The peak deceleration of the witness bunch is $290 \mathrm{keV}$ which is normalized to $0.44 \mathrm{MV} / \mathrm{m} / \mathrm{nC}$ over the $2.6 \mathrm{~cm}$ of the structure length. It indicates that $\sim 30 \mathrm{MV} / \mathrm{m}$ of peak gradient was reached while an $80 \mathrm{nC}$ electron bunch passing through the structure although the accelerating gradient was not clearly measured. No signatures of the rf breakdown were observed during the experiment at high charge (no distortion of the probe signal and no sparks were observed). Figure 6 shows the linear dependence of the signal strength of one monopole mode, obtained from the rf probe, with bunch charge. The charge was controlled by varying the laser intensity on the photocathode in the experiment.

In conclusion, we have experimentally studied fundamental and higher order modes of the wakefields in a PBG accelerating structure by excitation with a positioncontrolled beam. The results show that the $Q$ of the HOMs is significantly lower than that of the acceleration mode, which makes the PBG accelerating structure a compelling candidate for future accelerator design. The technique presented here can also be used to study wakefields in other geometrically complicated accelerating structures.

\section{ACKNOWLEDGMENTS}

This work was supported by the Department of Energy, High Energy Physics Division, under Contract No. DEAC02-06CH11357. We thank Huyu (Allen) Zhao, James Zmuda, and Marvin Lien for their work in building the stepping motor-driven translation stage.

[1] N. Kroll, D. R. Smith, and S. Schultz, in Proceedings of the 5th Advanced Accelerator Concepts Workshop, AIP Conf. Proc. No. 279 (AIP, Port Jefferson, NY, 1992), pp. 197-211.

[2] D. R. Smith, S. Schultz, N. Kroll, M. Sigalas, K. M. Ho, and C. M. Souloulis, Appl. Phys. Lett. 65, 645 (1994).

[3] C. Adolphsen, K. Bane, T. Higo, K. Kubo, R. Miller, R. Ruth, K. Thompson, and J. Wang, Phys. Rev. Lett. 74, 2475 (1995).

[4] Syratchev, Report No. CERN-AB-2005-086, CLIC Note 643 (unpublished).

[5] E. I. Smirnova, I. Mastovsky, M. A. Shapiro, and R. J. Temkin, Phys. Rev. ST Accel. Beams 8, 091302 (2005).

[6] S. L. McCall and P. M. Platzman, Phys. Rev. Lett. 67, 2017 (1991).

[7] M. R. Masullo, S. Andreone, E. Di Gennaro, S. Albanese, F. Francomacaro, M. Panniello, V. G. Vaccaro, and G. Lamura, Microw. Opt. Technol. Lett. 48, 2486 (2006).

[8] E. I. Smirnova, A. S. Kesar, I. Mastovsky, M. A. Shapiro, and R. J. Temkin, Phys. Rev. Lett. 95, 074801 (2005).

[9] R. A. Marsh, M.A. Shapiro, and R. J. Temkin, in Proceedings of the 2007 Particle Accelerator Conference, Albuquerque, New Mexico, 2007 (IEEE, Albuquerque, New Mexico, 2007), pp. 3002-3004.

[10] D. Li, N. Kroll, D. R. Smith, and S. Schultz, in Proceedings of the 4th Advanced Accelerator Concepts Workshop, AIP Conf. Proc. No. 398 (AIP, Lake Tahoe, CA, 1996), pp. 528-537.

[11] G. R. Werner, C. A. Bauer, and J. R. Cary, Phys. Rev. ST Accel. Beams 12, 071301 (2009).

[12] M. A. Shapiro, W. J. Brown, I. Mastovsky, J. R. Sirigiri, and R. J. Temkin, Phys. Rev. ST Accel. Beams 4, 042001 (2001).

[13] E. Di Gennaro, S. Savo, A. Andreone, V. Galdi, G. Castaldi, V. Pierro, and M. R. Masullo, Appl. Phys. Lett. 93, 164102 (2008).

[14] N. Kroll, S. Schultz, and D. R. Smith, and D. C. Vier, in Proceedings of the 18th Particle Accelerator Conference, New York, 1999 (IEEE, New York, 1999), pp. 830-832.

[15] C. Wu, Z. Li, R. Song, S. Dong, and X. He, in Proceedings of the International Conference on Microwave \& $\mathrm{mm}$ Wave Technology (ICMMT2008) (IEEE, New York, 2008), pp. 1576-1579.

[16] X. E. Lin, Phys. Rev. ST Accel. Beams 4, 051301 (2001).

[17] R. J. Noble, E. R. Colby, B. Cowan, C. M. Sears, and R. H. Siemann, Report No. SLAC-PUB-12571.

[18] K.-H. Jang, S.-Gy. Jeon, J.-II. Kim, J.-H. Won, J.-K. So, S.-H. Bak, A. Srivastava, S.-S. Jung, and G.-S. Park, Appl. Phys. Lett. 93, 211104 (2008).

[19] P. Xu, H. Chen, S. Zheng, F. Gao, W. Gai, J. Shi, and X. Guan, High Energy Phys. Nucl. Phys. 31, 678 (2007).

[20] www.hep.anl.gov/awa.

[21] E. I. Smirnova, C. Chen, M. A. Shapiro, and R. J. Temkin, in Proceedings of the 20th Particle Accelerator Conference, Portland, OR, 2003 (IEEE, New York, 2003), pp. 1258-1260. 